Research Article

\title{
Rapid Determination of Pemetrexed Concentration and Distribution in Human Breast Cancer Cells (McF-7) Based on UHPLC-MS/MS
}

\author{
Mengwei Zhang, ${ }^{1,2,3}$ Zhipeng Wang $(D),{ }^{2}$ Qiqiang Zhang, ${ }^{1}$ Qing Ye, ${ }^{1}$ Fengjing Xu, ${ }^{2}$ Lili Cui, ${ }^{2}$ \\ Yanping Liu, ${ }^{2}$ Shouhong Gao $\left(\mathbb{1},{ }^{2}\right.$ Wansheng Chen $\left(\mathbb{b},{ }^{2}\right.$ and Yan Liu $\mathbb{1}^{1}$ \\ ${ }^{1}$ Department of Pharmacy, Xinhua Hospital, Shanghai Jiao Tong University School of Medicine, Shanghai 200092, China \\ ${ }^{2}$ Department of Pharmacy, Changzheng Hospital, Second Military Medical University, Shanghai 200003, China \\ ${ }^{3}$ Key Laboratory for Research on Active Ingredients in Natural Medicine of Jiangxi Province, Yichun University, \\ Yichun 336000, China
}

Correspondence should be addressed to Shouhong Gao; gaoshouhong@smmu.edu.cn, Wansheng Chen; chenwansheng@smmu.edu.cn, and Yan Liu; liuyan03@xinhuamed.com.cn

Mengwei Zhang and Zhipeng Wang contributed equally to this work.

Received 13 December 2020; Revised 7 February 2021; Accepted 2 March 2021; Published 13 March 2021

Academic Editor: Barbara Bojko

Copyright (C) 2021 Mengwei Zhang et al. This is an open access article distributed under the Creative Commons Attribution License, which permits unrestricted use, distribution, and reproduction in any medium, provided the original work is properly cited.

\begin{abstract}
Cell is the basic unit of structure and function of all living bodies. The study of intracellular drug concentration distribution is helpful to understand the drug efficacy of target site. Pemetrexed is a new multitarget folate antagonist with pyrrolidine group as its core structure. An ultra high-performance liquid chromatography tandem mass spectrometry (UHPLC-MS/MS) method was developed for rapid quantification of pemetrexed concentration in human breast cancer cells (MCF-7). Sample pretreatment was completed by protein precipitation using methanol. The optimized chromatographic separation was achieved on a ZORBAX SB-C $\mathrm{C}_{18} \mathrm{column}(2.1 \times 150 \mathrm{~mm}$, $3.5 \mu \mathrm{m})$. The column was equilibrated with initial mobile phase and eluted under gradient phases containing $0.1 \%$ formic acid in water (phase A) and in acetonitrile (phase B). The gradient program started at 5\% B, increased to $95 \%$ B in 2 min, and then held at $95 \%$ B for $1.5 \mathrm{~min}$. The linear range of pemetrexed in cells and nucleus was $2.0-200.0 \mathrm{ng} / \mathrm{mL}$, while in the medium sample it was $50.0-5000.0 \mathrm{ng} /$ $\mathrm{ml}$, and the correlation coefficients were all greater than 0.99 . The recovery was $47.50-67.55 \%(2.0-200.0 \mathrm{ng} / \mathrm{mL})$ and $82.72-97.15 \%$ $(50.0-5000.0 \mathrm{ng} / \mathrm{mL})$, and the matrix effect was $98.10-100.62 \%(2.0-200.0 \mathrm{ng} / \mathrm{mL})$ and $89.78-97.65 \%(50.0-5000.0 \mathrm{ng} / \mathrm{mL}) . \mathrm{Interday}$ precision and intraday precision (RSD\%) were less than $15.0 \%$ (for LLOQ, less than $20 \%$ ), and accuracy (RE\%) was within $\pm 15 \%$; the deviation of stability was within $\pm 15 \%$, all meeting the requirements of biological sample analysis. The results of intracellular samples showed that the concentration of pemetrexed reached its peak at $3 \mathrm{~h}$ after administration. The concentration of pemetrexed in the nucleus continued to increase $2 \mathrm{~h}$ after administration and may have reached the maximum concentration at $6 \mathrm{~h}$.
\end{abstract}

\section{Introduction}

Breast cancer has become a serious disease endangering women's health all over the world. According to the 2020 global cancer report [1] released by the international agency for research on cancer, in 2018, the incidence of breast cancer in females ranked first among malignant tumors $(11.6 \%)$ and the mortality ranked fifth among malignant tumors (6.6\%). The National Cancer Report [2] 2019 shows that, in 2015, the incidence of breast cancer ranked the first among female malignant tumors in China, with about 304,000 new cases, and the mortality ranked the fifth among female malignant tumors (8.16\%), with both morbidity and mortality, showing an increasing trend in recent years.

At present, the treatment of breast cancer is still mainly through surgery and chemotherapy [3]. Efficacious chemotherapeutic regimen scan reduces the metastasis and recurrence, thereby increasing the possibility of extending 
survival time [4]. Nowadays, the chemotherapeutic agents included anthracycline, paclitaxol, platinum, and antimetabolism medicines, etc. [5]. Although impressive improvements have been made in adjuvant therapy with anthracyclines and taxanes in breast cancer, development of drug resistance to these agents in recurrent tumors is common [6-8], and there are still $20 \% \sim 30 \%$ of patients with primary drug resistance in clinical practice. Even if the initial treatment is effective, a vast majority of patients still have disease progression, and eventually develop metastatic disease. As a third-line chemotherapy agent, pemetrexed is a multitarget antimetabolite drug that inhibits folate metabolism and DNA synthesis enzymes. Response rates to pemetrexed as a single agent varied from $8 \%$ to $31 \%$, and in combination regimen which was reported between $15.8 \%$ and $55.7 \%$ [9]. Collective studies supported that pemetrexed effectively prolongs survival estimation in a proportion of advanced breast cancer patients, which was a better agent, especially followed with anthracycline- and taxane-containing regimens $[10-12]$.

Pemetrexed has good efficacy and compliance in patients with metastatic breast cancer. However, variable treatment response of pemetrexed chemotherapy was observed in different pathological types of tumors [13]. The concentration of pemetrexed in cells is considered to be an important factor affecting the efficacy. There is an urgent need for a dose optimization of pemetrexed for metastatic breast cancer that prolongs survival and improve tolerance. Studying the distribution of intracellular drug concentration is helpful to understand the drug efficacy in target sites, but there still is not a LC-MS/MS method reported until now. Thus, this study will develop an accurate and stable method based on UHPLC-MS/MS technology that can quickly determine the concentration and distribution of pemetrexed in cells, which is helpful for deep understanding of the drug efficacy of pemetrexed.

\section{Methods}

2.1. Chemicals. The standards of pemetrexed (Lot: D1103AS) and nilotinib (internal standard, IS) (Lot: D1201A) were purchased from Meilun Biotech Co., Ltd. (Dalian City, China). HPLC-grade methanol and acetonitrile were obtained from Merck (Merck Company, Darmstadt, Germany). HPLC-grade dimethyl sulfoxide (DMSO) was purchased from Tedia Company Inc (Tedia, Fairfield, OH, USA). HPLC-grade isopropanol was bought from Shanghai Titan technology Co., Ltd. (Titan, Shanghai, China). HPLCgrade formic acid was from Macklin biochemical Technology Co., Ltd. (Macklin, Shanghai, China). High glucose cell culture medium was supplied by Hyclone company (Logan, Utah, USA). Distilled water was purchased from Watsons Distilled Water Co. Ltd. (Watsons, Shenzhen, China). Human breast cancer McF-7 cell line was provided by Shanghai Branch of China Science Academy (Shanghai, China). Penicillin-Streptomycin solution was purchased from Shanghai Yuanye Bio-Technology Co., Ltd. (Yuanye, Shanghai, China). RIPA Lysis buffer was supplied from Shanghai Yuanye Biotechnology Co., Ltd. (Yuanye,
Shanghai, China). The cell nucleus extraction kit was got from Abbkine Scientific Co., Ltd. (Wuhan, China).

2.2. Instrumentation. All experiments were carried out on an Agilent 1290 series UHPLC system including an online degasser, a quatpump, an autosampler, and a column oven and interfaced to an Agilent $6460 \mathrm{~A}$ triplequadrupole mass spectrometer with an electrospray ionization source (ESI, Agilent Technologies, USA). All data were collected and analyzed through Agilent Masshunter workstation. Biosafety cabinet was from Thermo Scientific, Germany; inverted microscope was from Olympus, Tokyo, Japan; and temperature controlled magnetic agitator was from Abbott company, Zhengzhou, China.

2.3. Chromatographic Conditions. The ZORBAX SB-C18 column was selected as the analysis column of pemetrexed $(2.1 \times 150 \mathrm{~mm}, 3.5 \mu \mathrm{m}$, Agilent technology, USA). The mobile phase is acetonitrile and water (containing $0.1 \%$ formic acid). The mobile phase consisted of acetonitrile as organic phase (phase A) and water (containing $0.1 \%$ formic acid) as aqueous phase (phase B). The mobile phases were degassed automatically using the online degasser system, and the flow rate was $0.3 \mathrm{~mL} / \mathrm{min}$. The gradient program started at $5 \% \mathrm{~A}$, and increased to $95 \% \mathrm{~A}$ in $2 \mathrm{~min}$, and then was held at $95 \% \mathrm{~A}$ for $1.5 \mathrm{~min}$, the pastime was $2 \mathrm{~min}$, and the total run time was $5.5 \mathrm{~min}$. The column temperature was maintained at $35^{\circ} \mathrm{C}$, and the injection volume was set at $5 \mu \mathrm{L}$.

2.4. Mass Spectrum Parameters. In positive ion mode, ESI ion source was selected for mass spectrometry detection. Data scanning was performed in multiple reaction monitoring (MRM) mode (Figure 1). The dry gas, sheath gas, and nebulizer gas are all high pure nitrogen. The drying temperature was $325^{\circ} \mathrm{C}$, and the drying gas flow rate was $10 \mathrm{~L} /$ min. The temperature of sheath gas was $350^{\circ} \mathrm{C}$, and the flow rate of sheath gas was $12 \mathrm{~L} / \mathrm{min}$. The capillary voltage was $4000 \mathrm{~V}$ and the nebulizer pressure was $50 \mathrm{psi}$. The collision gas was high purity nitrogen and was set at $0.2 \mathrm{mPa}$. The main mass spectral parameters of pemetrexed and internal standard (IS, nilotinib) are shown in Table 1.

2.5. Preparation of Standard Solution. Accurately weighted $2.00 \mathrm{mg}$ pemetrexed was put into a $2 \mathrm{~mL}$ volumetric flask, and an appropriate amount of methanol was added to dissolve it with a few drops of DMSO. The stock solution was aliquoted and stored in a refrigerator at $-80^{\circ} \mathrm{C}$. The above-mentioned stock solution was diluted with $10 \%$ methanol to obtain a series of working solutions of the following concentrations: 20.0, 50.0, $100.0,200.0,500.0,1000.0,2000.0,5000.0,10000.0,20000.0$, $50000.0 \mathrm{ng} / \mathrm{mL}$ for pemetrexed. Two calibration curves were prepared and the working solution was diluted 10 times with blank medium to obtain the calibration sample; the concentration range of pemetrexed was $2.0-200.0 \mathrm{ng} / \mathrm{mL}$ and $50.0-5000.0 \mathrm{ng} / \mathrm{mL}$ for intracellular and extracellular samples, respectively. The quality control (QC) sample of pemetrexed is prepared separately in the same way. Finally, QC samples with 


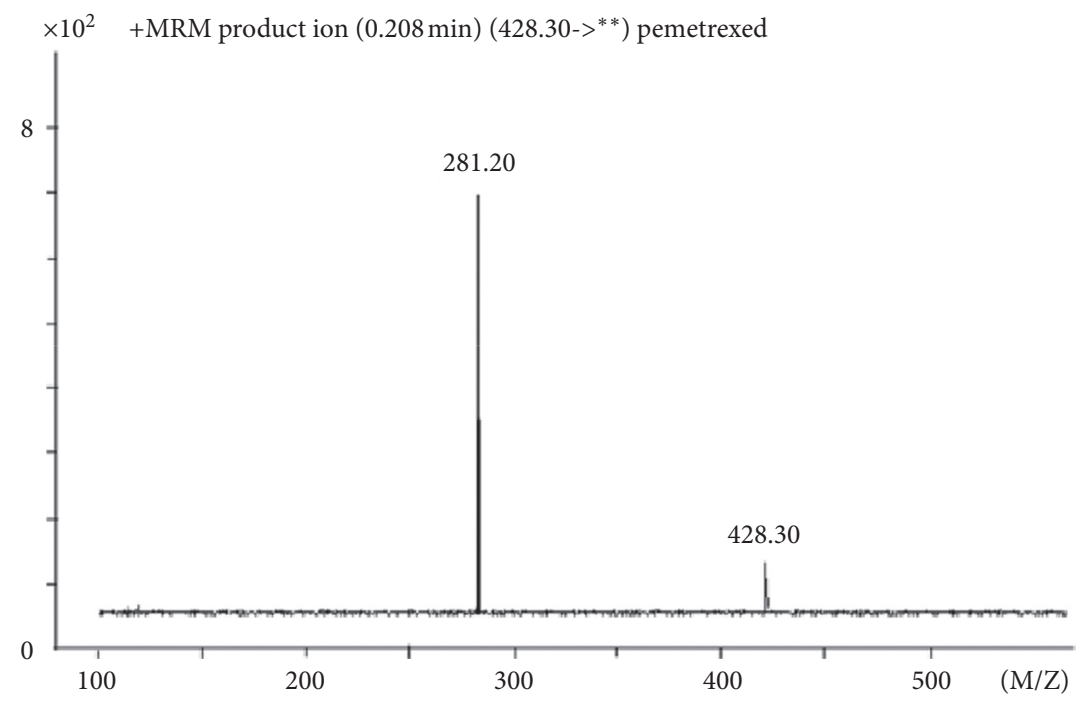

(a)

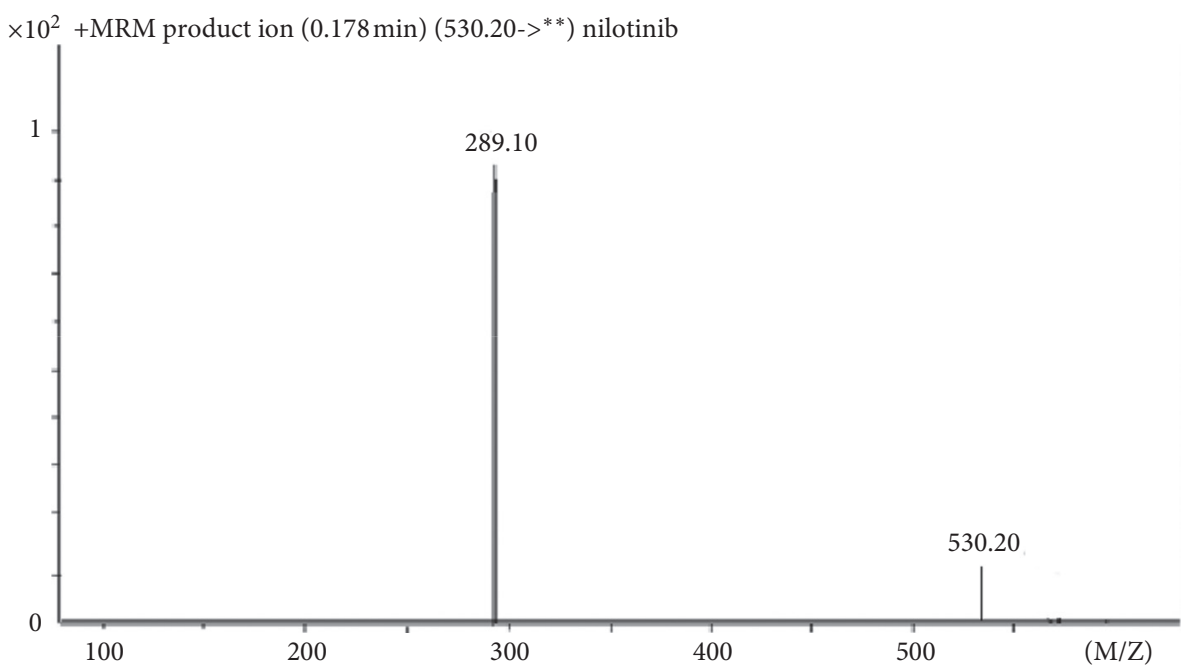

(b)

FIgUre 1: Product ions chromatograms and fragment structures of pemetrexed and nilotinib (IS). (a) Pemetrexed. (b) Nilotinib (IS).

TABLE 1: Main mass spectral parameters of pemetrexed and IS.

\begin{tabular}{lccccc}
\hline Name & Ionization type & Precursor ions $(\mathrm{m} / \mathrm{z})$ & Fragmentor $(\mathrm{V})$ & Collision energy $(\mathrm{eV})$ & Product ions $(\mathrm{m} / \mathrm{z})$ \\
\hline Pemetrexed & + & 428.3 & 80 & 10 & 281.2 \\
Nilotinib (IS) & + & 530.2 & 130 & 32 & 289.2 \\
\hline
\end{tabular}

concentration of $100.0,500.0,2000.0 \mathrm{ng} / \mathrm{mL}$ and $5.0,20.0$, $100.0 \mathrm{ng} / \mathrm{mL}$ were obtained. The IS solution was prepared using the same method, and $2 \mathrm{mg}$ nilotinib was dissolved in $2 \mathrm{~mL}$ methanol, and then diluted with methanol to obtain IS solutions with final concentrations of 2 and $20 \mathrm{ng} / \mathrm{mL}$, which were stored at $-20^{\circ} \mathrm{C}$ for later use.

2.6. Cell Culture. The human breast cancer cell line McF-7 was cultured with DMEM containing 10\% fetal bovine serum and double antibiotics $(100 \mu \mathrm{g} / \mathrm{ml}$ penicillin and $100 \mu \mathrm{g} / \mathrm{ml}$ streptomycin, complete medium) in incubator $\left(37^{\circ} \mathrm{C}, 5 \% \mathrm{CO}_{2}\right)$ and the medium was refreshed every 2 or 3 days. The cells were passaged or transferred to prepare stock solutions when they reach an $80-90 \%$ confluence.

\subsection{Sample Collection and Pretreatment}

2.7.1. Experiment Execution and Cell Samples Collection. When the cells grew to $90 \%$ confluence, the culture medium was discarded, and the cells were washed twice with $1 \times$ PBS. Pemetrexed solution in complete medium with a final concentration of $6 \mu \mathrm{g} / \mathrm{mL}$ was added to incubate the cells for $0,0.5$, 
$1,2,3$, and $6 \mathrm{~h}$, respectively. The culture medium was harvested as the medium sample (as a reference). After PBS wash, the cells were digested with $0.25 \%$ trypsin containing EDTA for cell detachment from the growth surface. The trypsin was neutralized with complete medium, and the mixture was transferred to a $15 \mathrm{~mL}$ centrifuge tube for centrifugation $(1000 \times \mathrm{g}, 5 \mathrm{~min}$, $4^{\circ} \mathrm{C}$ ). The supernatant was discarded and $100 \mu \mathrm{L}$ of RIPA lysate was added for full cleavage. The supernatant was collected as the intracellular sample after a centrifuge at $13400 \times \mathrm{g}$ for $5 \mathrm{~min}$. The experiments were carried out three times in triplicate using the same method. For the nuclear sample, a parallel assay was carried out and cells were cultured and treated using the same way, and the nuclear samples were collected after the pemetrexed treatment using an extraction kit.

2.7.2. Sample Pretreatment. Sample pretreatment was performed by ultrafiltration-protein precipitation method. An appropriate amount of culture medium sample was filtered through $0.22 \mu \mathrm{m}$ microporous filter membrane, and $60 \mu \mathrm{L}$ filtrate was transferred to a $1.5 \mathrm{~mL}$ Eppendorf tube prior to adding $340 \mu \mathrm{L}$ methanol (containing $20 \mathrm{ng} / \mathrm{mL}$ of IS solution). For intracellular and nuclear samples, $60 \mu \mathrm{L}$ filtrate was transferred to a $1.5 \mathrm{~mL}$ Eppendorf tube before adding $180 \mu \mathrm{L}$ methanol (containing $2 \mathrm{ng} / \mathrm{mL}$ of IS solution). After vortex mixing for $3 \mathrm{~min}$, the mixture was centrifuged at room temperature of $13000 \times \mathrm{g}$ for $10 \mathrm{~min}$. Then, $5 \mu \mathrm{L} \mathrm{su}-$ pernatant was directly injected for analysis.

2.8. Methodological Study. In order to ensure the accuracy, reliability, and stability of the determination results, the precision and accuracy, extraction recovery and matrix effect, stability, and specificity of this method should meet the requirements of biological sample analysis. Methodological verification was conducted in accordance with the Chinese pharmacopeia (version 2015) and FDA guidelines, and the experiments were carried out as we reported previously $[14,15]$.

\section{Results and Discussion}

3.1. Chromatography Condition Optimization. During the method development, several chromatographic columns had been tested, such as Zorbax SB-C ${ }_{18}$, Eclipse XDB-C ${ }_{18}$, Poroshell 120 SB- $\mathrm{C}_{18}$, Eclipse Plus- $\mathrm{C}_{18}$, etc. By comparing their chromatographic characteristics (resolution, peak time, response, peak shape, etc.), the results showed that the pemetrexed and IS had better peak shapes and responses on Zorbax $\mathrm{SB}-\mathrm{C}_{18}$ chromatographic column and could achieve complete separation from the internal interferent substances in a short time. Therefore, Zorbax SB- $\mathrm{C}_{18}$ column was selected for the development of methodology. Different mobile phase, acetonitrile and methanol, and additives including formic acid and ammonium acetate were selected to improve the peak shape, response, and retention time, but the methanol brought a longer retention time and trailing peak when ammonium acetate was added to the mobile phase, and the response of the analyte was low and the peak shape was asymmetrical. It was found that $0.1 \%$ formic acid (V:V) in the water phase could significantly increase the response of the analyte, and $0.1 \%$ formic acid $(\mathrm{V}: \mathrm{V})$ in the organic phase has a similar effect on the response of analyte [16], so a $0.1 \%$ formic acid was simultaneously added to the water and organic phase to achieve a better response of pemetrexed in the method development. In order to shorten the analysis time and optimize the gradient of the mobile phase, we slightly accelerated the increase of organic phase which could shorten the retention time of the analyte, because a quick increase of organic phase would lead to a worse peak shape of the analyte. In general, improving the ratio of the organic phase in the initial mobile phase could reduce the retention time of most analytes, but in our experiment, a higher ratio of acetonitrile damaged the symmetry of peak, so the total analytical time was $5 \mathrm{~min}$ and the ratio of organic phase was set to $5 \%$ in the initial mobile phase.

3.2. Sample Pretreatment. Sample pretreatment is an important step in the process of analytes extraction. The process of sample treatment has a significant impact on the experimental results, for example, reducing the matrix effect, which is indispensable to improving the precision and accuracy. Therefore, a further improvement of the resolution and sensitivity of the chromatographic system requires a more suitable sample pretreatment method. When a sample has relatively high purity and concentration of the analyte, a simpler pretreatment could meet the analyte extraction; otherwise, a complex extraction process, for instance, adsorption and enrichment, etc., is needed [17]. The main purpose of sample pretreatment is to remove proteins, some lipids, and soluble salts. At present, protein precipitation, solid-phase extraction, and liquid-liquid extraction are commonly used for sample pretreatment, and every method has its own advantages and disadvantages. The protein precipitation method is simple, fast, and economical, but there are still many impurities in the matrix after treatment, which usually bring a strong matrix effect. The sample processed using liquid-liquid extraction is clean, but the extraction recovery largely depends on the physicochemical property of analytes and the cost is higher than protein precipitation. The solid-phase extraction method often presents a stable extraction rate and clean matrix, but the cost is highest in the three methods and a sophisticated skill was necessary to gain a stable recovery. By comparing the extraction recovery and matrix effect of methanol and acetonitrile in different proportion, the results showed a higher extraction recovery when methanol was applied as precipitant and the ratio of methanol to sample is $3: 1$. In order to further purify the sample and reduce the contamination of cell debris in the sample, we first filtered the sample with $0.22 \mu \mathrm{m}$ microporous membrane and then precipitated the protein in the filtrate with methanol. The ultrafiltration combined with protein precipitation method is more suitable in this experiment.

\subsection{Method Validation}

3.3.1. Specificity. By comparing the chromatograms of blank samples, IS, LLOQ, and measured samples (Figure 2), there were no interfering substances in the same retention time of pemetrexed, indicating that this method has good specificity and meets the relevant requirements. 

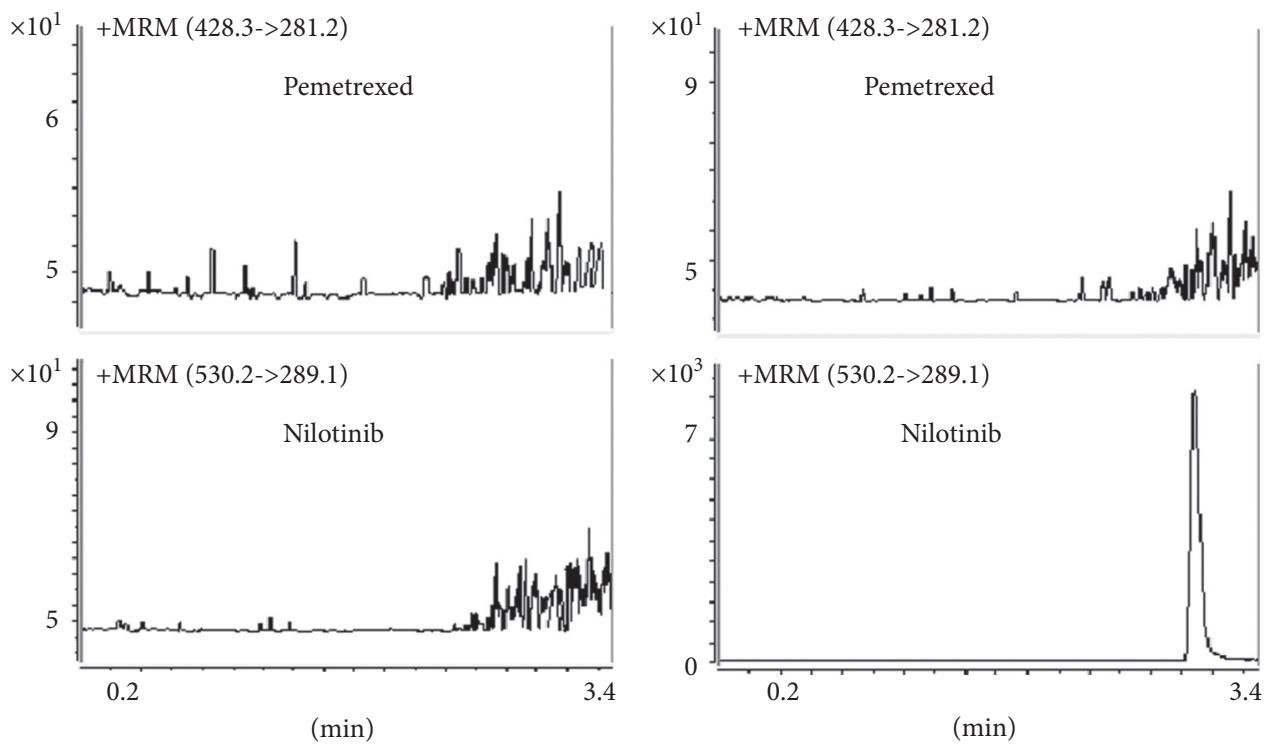

(a)

(b)

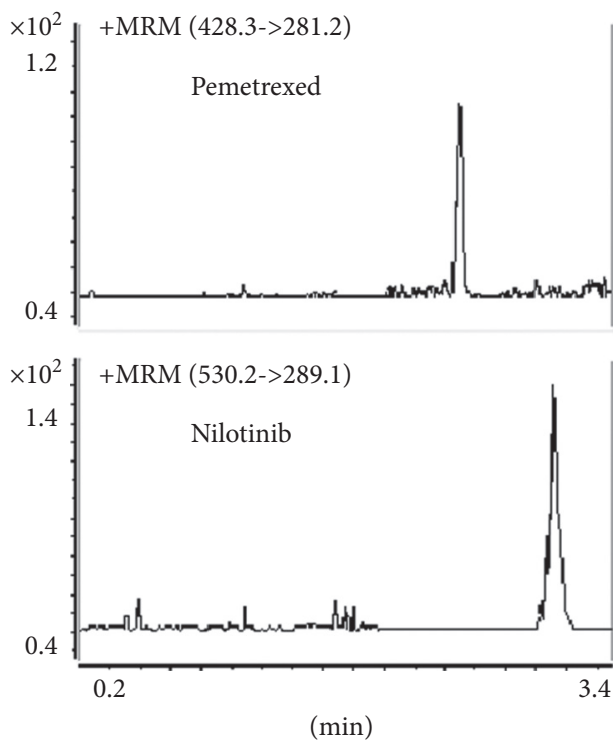

(c)
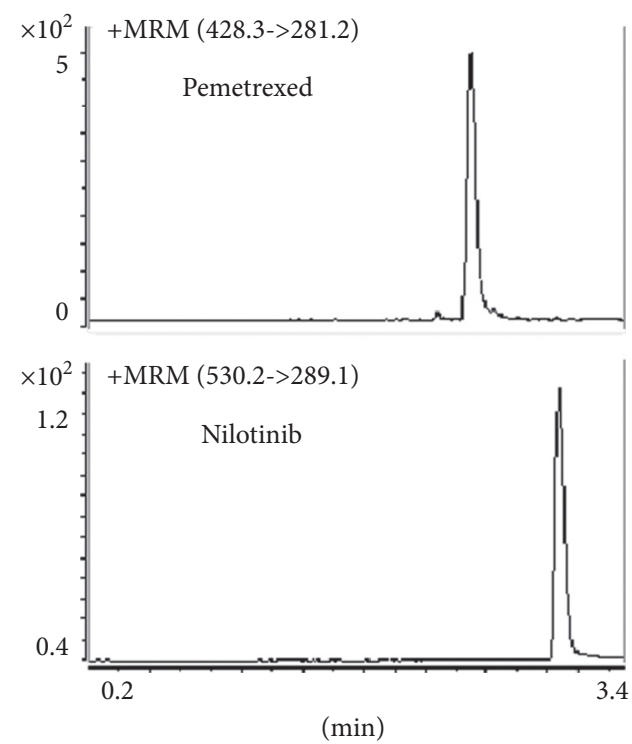

(d)

FiguRE 2: Chromatograms of pemetrexed and IS in different samples. (a) Blank sample. (b) Blank sample spiked with IS. (c) Blank sample spiked with LLOQ concentration of pemetrexed. (d) Real sample.

3.3.2. Linearity of Calibration Curves and LLOQ. The linear range of intracellular samples was $2-200 \mathrm{ng} / \mathrm{mL}$ and that of extracellular samples was $50-5000 \mathrm{ng} / \mathrm{mL}$. The results of intracellular sample calibration curves are shown in Table 2. The results show that the regression type of correction curve is linear, the weight factor is $1 / \chi^{2}$, and the linear correlation coefficient $R^{2}$ is greater than 0.99 , which indicates that the correction curve has a good linear relationship and meets the requirements of correlation analysis. The LLOQ for intracellular samples was $2 \mathrm{ng} / \mathrm{ml}$. The carryover of pemetrexed that needed to be investigated after the method was developed. After the calibration sample with the highest concentration of pemetrexed was detected, the blank sample was then tested, repeating for 3 cycles to observe the response of pemetrexed and IS in the blank sample. Compared with the LLOQ sample, the response of pemetrexed in the blank sample was less than $20 \%$ of that in LLOQ sample, and the response of IS in the blank sample was less than $5 \%$ of that in LLOQ sample (Figure 3) (the methodological data for the other calibration curve of 50-5000 ng/ $\mathrm{ml}$ are shown in Supplementary 1).

3.3.3. Interprecision and Intraprecision and Accuracy. QC samples of three concentrations (low, middle, and high) and LLOQ were selected to investigate the precision and accuracy of the method. The results of intraday and interday precision and accuracy of pemetrexed are shown in Table 3. 
TABLE 2: Linearity regression parameters of pemetrexed.

\begin{tabular}{lccccc}
\hline Analyte & Regression type & Linear range & Weighing factor & Regression equations & $R^{2}$ \\
\hline Pemetrexed & Linearity & $2-200 \mathrm{ng} / \mathrm{mL}$ & $1 / x^{2}$ & $Y=0.003429 * x+0.001325$ & 0.998 \\
\hline
\end{tabular}

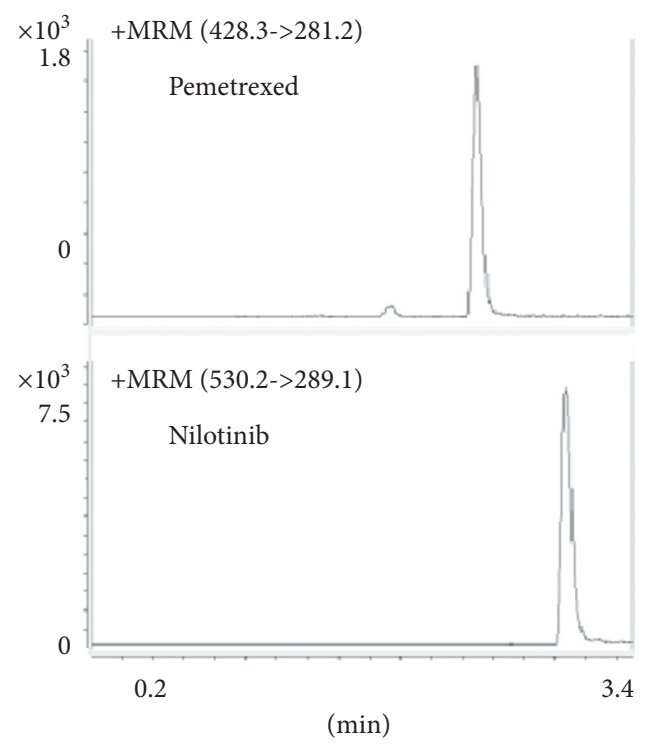

(a)

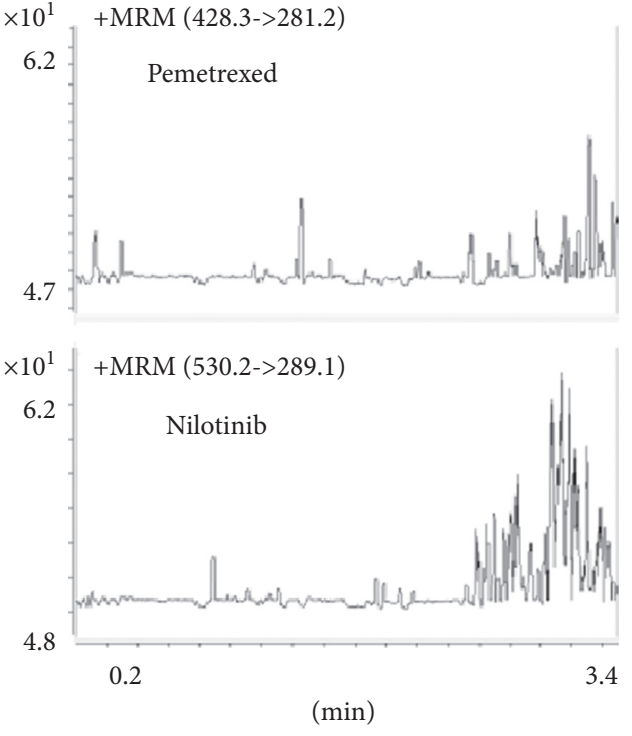

(b)

FIgURE 3: The results of carryover of pemetrexed. (a) Highest calibration standard sample and (b) following blank sample.

TABLE 3: Interprecision and intraprecision and accuracy of pemetrexed $(n=5)$.

\begin{tabular}{|c|c|c|c|c|c|c|c|}
\hline \multirow[b]{2}{*}{ Analyte } & \multirow[b]{2}{*}{$\begin{array}{c}\text { Nominal } \\
\text { concentration } \\
(\mathrm{ng} / \mathrm{mL})\end{array}$} & \multicolumn{3}{|c|}{ Intraday } & \multicolumn{3}{|c|}{ Interday } \\
\hline & & $\begin{array}{c}\text { Measured } \\
\text { concentration }(\mathrm{ng} / \\
\mathrm{mL}, \text { mean } \pm \mathrm{SD})\end{array}$ & $\begin{array}{c}\text { Precision } \\
(\mathrm{RSD} \%)\end{array}$ & $\begin{array}{c}\text { Accuracy } \\
\text { (RE\%) }\end{array}$ & $\begin{array}{c}\text { Measured } \\
\text { concentration }(\mathrm{ng} / \\
\mathrm{mL}, \text { mean } \pm \mathrm{SD})\end{array}$ & $\begin{array}{c}\text { Precision } \\
\text { (RSD\%) }\end{array}$ & Accuracy (RE\%) \\
\hline \multirow{4}{*}{ Pemetrexed } & 2 & $1.74 \pm 0.40$ & 14.12 & 92.49 & $1.70 \pm 0.28$ & 14.67 & 85.19 \\
\hline & 5 & $4.84 \pm 0.65$ & 13.43 & 96.78 & $4.50 \pm 0.60$ & 13.40 & 90.08 \\
\hline & 20 & $19.15 \pm 0.70$ & 3.63 & 95.76 & $17.34 \pm 1.58$ & 9.09 & 86.71 \\
\hline & 100 & $100.51 \pm 3.14$ & 3.12 & 100.51 & $98.21 \pm 2.94$ & 2.99 & 98.21 \\
\hline
\end{tabular}

The results show that the intraday precision and interday precision (RSD\%) of pemetrexed were below $14.67 \%$; the accuracy (RE\%) of pemetrexed in the low, middle, and high QC samples was within $\pm 15 \%$, and the accuracy (RE\%) of pemetrexed in the LLOQ samples was within $\pm 20 \%$, showing this method has good precision and accuracy.

3.3.4. Extraction Recovery and Matrix Effect. In this method, the extraction recovery and matrix effect results of pemetrexed are shown in Table 4. The results show that the extraction recovery of pemetrexed was between $47.50 \%$ and $67.55 \%$, and the matrix effect was between $98.10 \%$ and $100.62 \%$. In addition, the RSD of pemetrexed extraction recovery and matrix effect were less than $15 \%$, which indicated that the extraction recovery and matrix effect of pemetrexed were stable.

3.3.5. Stability. Stability investigation includes long-term stability, short-term stability, and freeze-thaw stability.
Long-term stability refers to the stability of the QC sample after 3 months in a refrigerator at $-80^{\circ} \mathrm{C}$; short-term stability is the stability of the QC sample after being placed in the automatic sampler at $4^{\circ} \mathrm{C}$ for 12 hours; freeze-thaw stability was assessed using QC sample after three freeze-thaw cycles. The stability of pemetrexed under different conditions was investigated at low and high concentration and the results are shown in Table 5 . The results show that the accuracy (RE $\%$ ) of pemetrexed ranged from $87.35 \%$ to $96.80 \%$, indicating that pemetrexed was stable in the conditions mentioned above.

3.4. The Concentration of Pemetrexed in McF-7 Cell and Medium. The measured results of culture medium samples, intracellular samples, and nuclear samples are shown in Table 6. The results of cell samples show that the concentration of pemetrexed in cells reached its peak at $3 \mathrm{~h}$ after administration. The concentration of pemetrexed in the nucleus continued to increase $2 \mathrm{~h}$ after administration and 
TABLE 4: The extraction recovery and matrix effect results of pemetrexed.

\begin{tabular}{|c|c|c|c|c|c|}
\hline \multirow{2}{*}{ Analyte } & \multirow{2}{*}{ Spiked concentration $(\mathrm{ng} / \mathrm{mL})$} & \multicolumn{2}{|c|}{ Recovery } & \multicolumn{2}{|c|}{ Matrix effect } \\
\hline & & Mean $(\%) \pm S D$ & RSD (\%) & Mean $(\%) \pm S D$ & RSD (\%) \\
\hline \multirow{2}{*}{ Pemetrexed } & 5 & $63.55 \pm 0.12$ & 9.25 & $98.10 \pm 0.05$ & 5.32 \\
\hline & 100 & $47.50 \pm 0.01$ & 1.70 & $100.62 \pm 0.01$ & 1.31 \\
\hline
\end{tabular}

TABLE 5: Results of stability investigation of pemetrexed $(n=6)$.

\begin{tabular}{|c|c|c|c|c|c|c|c|}
\hline \multirow[b]{2}{*}{ Analyte } & \multirow[b]{2}{*}{$\begin{array}{c}\text { Nominal } \\
\text { concentration } \\
(\mathrm{ng} / \mathrm{mL})\end{array}$} & \multicolumn{2}{|c|}{ Freeze-thaw stability } & \multicolumn{2}{|c|}{$24 \mathrm{~h}$ in autosampler } & \multicolumn{2}{|c|}{ Long-term stability (3 months) } \\
\hline & & $\begin{array}{c}\text { Measured } \\
\text { concentration } \\
(\mathrm{ng} / \mathrm{mL})\end{array}$ & $\begin{array}{c}\text { Accuracy } \\
\text { (RE\%) }\end{array}$ & $\begin{array}{c}\text { Measured } \\
\text { concentration } \\
(\mathrm{ng} / \mathrm{mL})\end{array}$ & $\begin{array}{c}\text { Accuracy } \\
(\mathrm{RE} \%)\end{array}$ & $\begin{array}{c}\text { Measured } \\
\text { concentration } \\
(\mathrm{ng} / \mathrm{mL})\end{array}$ & $\begin{array}{c}\text { Accuracy } \\
\text { (RE\%) }\end{array}$ \\
\hline & 5 & 4.52 & 90.40 & 4.84 & 96.80 & 4.47 & 89.40 \\
\hline Pemetrexe & 100 & 88.29 & 88.29 & 89.48 & 89.48 & 87.35 & 87.35 \\
\hline
\end{tabular}

TABLE 6: The concentration variations of pemetrexed in medium, cytoplasm, and cell nucleus $(n=3$, mean \pm SD).

\begin{tabular}{lccc}
\hline Time $(\mathrm{h})$ & Intracellular sample concentration $(\mathrm{ng} / \mathrm{mL})$ & Nuclear sample concentration $(\mathrm{ng} / \mathrm{mL})$ & Medium sample concentration $(\mathrm{ng} / \mathrm{mL})$ \\
\hline 0 & $\mathrm{ND}$ & $\mathrm{ND}$ & ND \\
0.5 & $4.24 \pm 0.53$ & $3.60 \pm 0.04$ & $1689.69 \pm 83.79$ \\
1 & $7.45 \pm 2.53$ & $4.25 \pm 0.01$ & $1793.39 \pm 18.75$ \\
2 & $19.94 \pm 1.26$ & $6.52 \pm 0.34$ & $1742.55 \pm 24.40$ \\
3 & $41.2 \pm 0.76$ & $4.98 \pm 1.87$ & $1741.00 \pm 42.47$ \\
6 & $35.32 \pm 0.64$ & $29.10 \pm 0.66$ & $1468.20 \pm 43.84$ \\
\hline
\end{tabular}

ND: not detected.

reached the maximum concentration at $6 \mathrm{~h}$. The pemetrexed was usually administrated with $500 \mathrm{mg} / \mathrm{m}^{2}$ as a third-line chemotherapeutic drug in breast cancer treatment on day 1 in a three-week course. Both the uptake and elimination process in cancer cell had certain influence on the intracellular concentration. Some research reported that the overexpression of TP-binding cassette (ABC) superfamily, including $\mathrm{P}$-glycoproteins (P-gp/ABCB1), breast cancer resistance proteins (BCRP, also named ABCG2), and ABCC subfamily (such as ABCC1-12), significantly decreased the intracellular concentration of pemetrexed based on accelerated drug efflux [18-20], which was an important factor for drug resistance of breast cancer to pemetrexed [21]. Our results presented the concentration variation of pemetrexed in McF-7 cells, which may be a hint to drug combination and dose optimization to achieve a synergistic effect, and finally a better response.

\section{Conclusion}

In this study, a sensitive and reliable UHPLC-MS/MS method was established, which can quickly determine the content of pemetrexed in cells. The pretreatment method, ultrafiltration-protein precipitation, is simple, rapid, and economical for the pemetrexed extraction for from cells, and the results presented a good specificity. The linear range, LLOQ, extraction recovery, matrix effect, interday and intraday precision and accuracy, stability, and so on of this method all met the requirements of biological sample analysis. The results of cell samples showed that the concentration of pemetrexed reached its peak $3 \mathrm{~h}$ after administration. The concentration of pemetrexed in the nucleus increased from $2 \mathrm{~h}$ to $6 \mathrm{~h}$ and may continue to accumulate inside the nucleus. Further study about the concentration and distribution of pemetrexed in cells will be helpful to understand the therapeutic effect of pemetrexed.

\section{Data Availability}

All data are included within in the article and the supplementary materials.

\section{Conflicts of Interest}

The authors declare that they have no conflicts of interest.

\section{Authors' Contributions}

Mengwei Zhang and Zhipeng Wang contributed equally to this work.

\section{Acknowledgments}

This work was supported by the National Institute of Chinese Natural Science Foundation (grant no. 81202598) and Shanghai Municipal Bureau of Health (grant no. 20ZR1435400) to Y. L.

\section{Supplementary Materials}

The methodological data for the other calibration curve of 50-5000 ng/ml are shown in Supplementary Materials. Figure 1: representative MRM chromatograms of pemetrexed. (a) Blank sample; (b) blank sample spiked IS; (c) blank sample spiked with LLOQ concentration of 
pemetrexed; (d) real sample. Table 1: linearity regression parameters of pemetrexed. Figure 2: comparative chromatograms of carryover of pemetrexed. (a) Highest calibration standard sample and (b) blank sample. Table 2: interprecision and intraprecision and accuracy of pemetrexed $(n=5)$. Table 3: recovery and matrix effect of pemetrexed (\%). Table 4: results of stability investigation of pemetrexed. (Supplementary Materials)

\section{References}

[1] C. P. Wild, E. Weiderpass, and B. W. Stewart, Cancer Research for Cancer Prevention, World Cancer Reports, International Agency for Research on Cancer, Lyon, France, 2020, http:// publications.iarc.fr/586.

[2] R. S. Zheng, K. X. Sun, S. W. Zhang et al., "An analysis of the prevalence of malignant tumors in China in 2015," Chinese Journal of Oncology, vol. 43, no. 1, pp. 19-28, 2019.

[3] F. Bai, Y. Yin, T. Chen et al., "Development of liposomal pemetrexed for enhanced therapy against multidrug resistance mediated by ABCC5 in breast cancer," International Journal of Nanomedicine, vol. 13, pp. 1327-1339, 2018.

[4] S. H. A. N. Fei, L. I. U. Yu-Lin, Q. I. A. N. G. Wang et al., "Thymidylate synthase predicts poor response to pemetrexed chemotherapy in patients with advanced breast cancer," Oncology Letters, vol. 16, pp. 3274-3280, 2018.

[5] Q.-Q. Deng, X.-E. Huang, L.-H. Ye, Y.-Y. Lu, Y. Liang, and J. Xiang, "Phase II trial of loubo (lobaplatin) and pemetrexed for patients with metastatic breast cancer not responding to anthracycline or taxanes," Asian Pacific Journal of Cancer Prevention, vol. 14, no. 1, pp. 413-417, 2013.

[6] A. Sheri and S. Johnston, "New developments and future directions in systemic therapy," Clinical Oncology, vol. 25, no. 2, pp. 117-126, 2013.

[7] V. Kataja and M. Castiglione, "Locally recurrent or metastatic breast cancer: ESMO Clinical Recommendations for diagnosis, treatment and follow-up," Annals of Oncology, vol. 19, no. suppl 2, pp. iil1-ii13, 2008.

[8] D. M. Parkin, F. Bray, J. Ferlay, and P. Pisani, "Global cancer statistics, 2002," CA: A Cancer Journal for Clinicians, vol. 55, no. 2, pp. 74-108, 2005.

[9] L.-Y. Zhou, Y.-H. Shi, Y.-S. Jia et al., "Potential role of pemetrexed in metastatic breast cancer patients pretreated with anthracycline or taxane," Chronic Diseases and Translational Medicine, vol. 23, no. 1, pp. 27-35, 2015.

[10] A. Garin, A. Manikhas, M. Biakhov et al., "A phase II study of pemetrexed and carboplatin in patients with locally advanced or metastatic breast cancer," Breast Cancer Research and Treatment, vol. 110, no. 2, pp. 309-315, 2008.

[11] A. Llombart-Cussac, M. Theodoulou, K. Rowland et al., "Pemetrexed in patients with locally advanced or metastatic breast cancer who had received previous anthracycline and taxane treatment: phase II study," Clinical Breast Cancer, vol. 7, no. 5, pp. 380-385, 2006.

[12] C. Dittrich, E. Solska, A. Manikhas et al., "A phase II multicenter study of two different dosages of pemetrexed given in combination with cyclophosphamide as first-line treatment in patients with locally advanced or metastatic breast cancer," Cancer Investigation, vol. 30, no. 4, pp. 309-316, 2012.

[13] N. J. Robert, P. R. Conkling, M. A. O’Rourke et al., "Results of a phase II study of pemetrexed as first-line chemotherapy in patients with advanced or metastatic breast cancer," Breast
Cancer Research and Treatment, vol. 126, no. 1, pp. 101-108, 2011.

[14] Z. Wang, Y. Yang, F. Zhang et al., "A direct, sensitive and efficient method for determination of alpha-fluoro-beta-alanine in urine: evaluating the influence of magnesium isoglycyrrhizinate on excretion in rat model," Journal of Chromatography B, vol. 1102-1103, pp. 17-22, 2018.

[15] S. Gao, Z. Tao, J. Zhou et al., "One-step solid extraction for simultaneous determination of eleven commonly used anticancer drugs and one active metabolite in human plasma by HPLC-MS/MS," Journal of Analytical Methods in Chemistry, vol. 2018, Article ID 7967694, 12 pages, 2018.

[16] J. Zhou, S. Gao, F. Zhang et al., "Liquid chromatographytandem mass spectrometry method for simultaneous determination of seven commonly used anticancer drugs in human plasma," Journal of Chromatography B: Analytical Technologies in the Biomedical and Life Sciences, vol. 1, pp. 1-8, 2012.

[17] Y. Liu, Z. Rong, X. Dong et al., "Detection technologies and metabolic profiling of bile acids: a comprehensive review," Lipids in Health and Disease, vol. 17, p. 121, 2018.

[18] M. M. Gottesman, T. Fojo, and S. E. Bates, "Multidrug resistance in cancer: role of ATP-dependent transporters," Nature Reviews Cancer, vol. 2, no. 1, pp. 48-58, 2002.

[19] A. W. Ravna, I. Sylte, and G. Sager, "A molecular model of a putative substrate releasing conformation of multidrug resistance protein 5 (MRP5)," European Journal of Medicinal Chemistry, vol. 43, no. 11, pp. 2557-2567, 2008.

[20] M. Dean, T. Fojo, and S. Bates, "Tumour stem cells and drug resistance," Nature Reviews Cancer, vol. 5, no. 4, pp. 275-284, 2005.

[21] J. Liang, T. Lu, Z. Chen, C. Zhan, and Q. Wang, "Mechanisms of resistance to pemetrexed in non-small cell lung cancer," Translational Lung Cancer Research, vol. 8, no. 6, pp. 11071118, 2019. 